\title{
Hearing Without Listening: Functional Connectivity Reveals the Engagement of Multiple Nonauditory Networks During Basic Sound Processing
}

\author{
Dave R.M. Langers ${ }^{1-3}$ and Jennifer R. Melcher ${ }^{1,4,5}$
}

\begin{abstract}
The present functional magnetic resonance imaging (fMRI) study presents data challenging the traditional view that sound is processed almost exclusively in the classical auditory pathway unless imbued with behavioral significance. In a first experiment, subjects were presented with broadband noise in on/off fashion as they performed an unrelated visual task. A conventional analysis assuming predictable sound-evoked responses demonstrated a typical activation pattern that was confined to classical auditory centers. In contrast, spatial independent component analysis (sICA) disclosed multiple networks of acoustically responsive brain centers. One network comprised classical auditory centers, but four others included nominally "nonauditory" areas: cingulo-insular cortex, mediotemporal limbic lobe, basal ganglia, and posterior orbitofrontal cortex, respectively. Functional connectivity analyses confirmed the sICA results by demonstrating coordinated activity between the involved brain structures. In a second experiment, fMRI data obtained from unstimulated (i.e., resting) subjects revealed largely similar networks. Together, these two experiments suggest the existence of a coordinated system of multiple acoustically responsive intrinsic brain networks, comprising classical auditory centers but also other brain areas. Our results suggest that nonauditory centers play a role in sound processing at a very basic level, even when the sound is not intertwined with behaviors requiring the well-known functionality of these regions.
\end{abstract}

Key words: central auditory system; functional connectivity; functional magnetic resonance imaging (fMRI); intrinsic brain networks; resting state; sound processing

\section{Introduction}

A COUStic Stimulation is well known to induce neural activity in the classical auditory pathway. This comprises the auditory nerve, various nuclei in the brainstem, midbrain, and thalamus, and multiple cortical areas in the superior surface of the temporal lobe (Ehret, 1997). However, microelectrode recordings in animals as well as humans are clear in showing that sound also activates neurons in other brain regions, such as frontal cortex, striatum, hippocampus, and amygdala (Boutros et al., 2005; Newman and Lindsley, 1976; Wilson et al., 1984). In some cases, the sound stimuli shown to engage these "nonauditory" regions were meaningful (e.g., species-specific vocalizations) or incorporated into a task requiring cognitive functions typically assigned to the recording location (e.g., memory, emotional processing, motor planning). In others, however, the sound stimuli were simple (e.g., clicks, tones, broadband noise) and presented during passive listening. The latter observations indicate that even rudimentary sounds lacking any obvious behavioral significance can elicit neuronal responses in nominally "nonauditory" brain areas.

Yet, neuroimaging studies rarely report activation outside the classical auditory system during passive listening to simple sounds or during a "minimal load" task on such sounds. This may be attributed to a scarcity of acoustically responsive neurons in nonauditory areas, resulting in a population response that is insufficiently large to be detectable. However,

\footnotetext{
${ }^{1}$ Eaton-Peabody Laboratory, Massachusetts Eye and Ear Infirmary, Boston, Massachusetts.

${ }^{2}$ Department of Otorhinolaryngology, University Medical Center Groningen, Groningen, The Netherlands.

${ }^{3}$ Faculty of Medical Sciences, School of Behavioral and Cognitive Neurosciences, University of Groningen, Groningen, The Netherlands.

${ }^{4}$ Department of Otology and Laryngology, Harvard Medical School, Boston, Massachusetts.

${ }^{5}$ Speech and Hearing Biociences and Technology Program, Harvard-MIT Division of Health Sciences and Technology, Massachusetts Institute of Technology, Cambridge, Massachusetts.
} 
alternatively, neural engagement by sound could have occurred widely throughout nonauditory areas, but it may have been missed, because it does not conform to the assumptions of most imaging analyses. The present study investigated this latter possibility.

In a first experiment, we used functional magnetic resonance imaging (fMRI) to test for sound-evoked activity in neural populations outside the classical auditory pathway during repeated presentation of an unattended meaningless broadband noise stimulus. Conventional methods that assume predictable responses to sound stimulation were used to verify the usual absence of activation outside of the classical auditory centers. In addition, functional connectivity methods free of any assumptions about the relationship between response and stimulus were applied. By means of spatial independent component analysis (sICA), multiple brain networks were extracted. Each was then tested for soundrelated activity. Correlation analyses were carried out to assess the coordination of activity between brain structures comprising networks found to contain sound-related activity. Finally, based on the assumption that passive hearing will also occur during rest, fMRI data were obtained from unstimulated subjects in a second experiment to test whether overt sound stimulation was required for the coordination of activity across brain centers. We hypothesized that widespread sound-evoked brain activity would be detectable outside the classical auditory system, provided that the common assumption that neural activity faithfully follows acoustic stimulation is relaxed.

\section{Materials and Methods}

\section{Subjects}

Sixteen subjects ( 9 men, 7 women; age: $25-59$ years, mean: 46 years; 15 right-handed, 1 ambidextrous) were invited to participate in this study on the basis of written informed consent, in approved accordance with the institutional committees on the use of human subjects at the Massachusetts Eye and Ear Infirmary, the Massachusetts General Hospital, and the Massachusetts Institute of Technology. Subjects did not have any known history of auditory, neurological, or psychiatric disorders. Audiometry was performed to confirm that all subjects had normal hearing: pure-tone thresholds did not exceed $25 \mathrm{~dB}$ HL at all standard audiometric frequencies in a range of $250-8000 \mathrm{~Hz}$.

\section{Stimuli and task}

Subjects performed a simple visual task while simultaneously being presented with unrelated acoustic stimuli.

On a uniformly lit background, a black cross was displayed that briefly turned red at pseudo-random intervals (approximately eight times per minute). Subjects were instructed to press a button device when they observed such a color change. Responses were monitored to ensure that subjects understood the task and stayed alert. The achieved hit rate typically equaled $80 \%-90 \%$.

At the same time, MR-compatible piezo-electric speakers that were mounted in insert ear plugs were used to deliver 41/2-min-long runs of auditory stimulation. Each run consisted of four 32-sec segments of broadband noise, separated by periods of silence of approximately equal duration ( $\mathrm{Gu}$ et al.,
2010). Noise intensity was constant throughout a run at 50 , 70, or $80 \mathrm{~dB}$ SPL. Between 5 and $11 \mathrm{fMRI}$ runs were carried out per subject, resulting in 132 runs in total.

In six subjects, 5 to 6 rest runs were additionally acquired, resulting in 31 runs in total. These differed only from the previously described runs with regard to a complete absence of audiovisual stimulus presentations or task performance. Instead, subjects were instructed only to lie still with their eyes open.

\section{Functional imaging}

Subjects lay supinely in the bore of a 3.0-T MR system that was equipped with a 12-channel head coil (Siemens Trio with Matrix head coil). A sagittal $T_{1}$-weighted MPRAGE scan was acquired for anatomical orientation (repetition time [TR]: $2530 \mathrm{msec}$; echo time [TE]: $3.39 \mathrm{msec}$; inversion time: 1100 msec; flip angle: $7^{\circ}$; matrix: $256 \times 256 \times 128$; voxel dimensions: $1.0 \times 1.0 \times 1.3 \mathrm{~mm}^{3}$ ). On these high-resolution images, an oblique coronal imaging volume was positioned parallel to the plane through the cochlear nuclei and inferior colliculi. The volume contained the subcortical nuclei of the classical auditory pathway, and by extending anteriorly toward the temporal pole, it encompassed at least the expanse of superior temporal lobe typically activated by rudimentary sound stimuli like the one used here. Functional scans were acquired in this partial brain volume and consisted of a dynamic series of $\mathrm{T}_{2}{ }^{*}$-sensitive echo planar imaging sequences (TR: $\sim 8 \mathrm{sec}$; TE: 30 msec; flip angle: $90^{\circ}$; matrix: $64 \times 64 \times 10$; voxel dimensions: $3.1 \times 3.1 \times 8.0 \mathrm{~mm}^{3}$, including $2.0 \mathrm{~mm}$ slice gap). Each functional imaging run consisted of 36 volume acquisitions, excluding initial startup scans that were rejected before the analysis. A sparse clustered volume scanning paradigm was employed to reduce the influence of acoustic scanner noise (Edmister et al., 1999; Hall et al., 1999). The scanner coolant pump and patient fan were turned off during functional imaging to further diminish ambient noise levels. Cardiac triggering on the basis of a pulse-oximeter signal was used to reduce the influence of cardiac motion (Guimaraes et al., 1998). In one subject, the cardiac signal was too weak to detect reliably, and a fixed TR of $7.5 \mathrm{sec}$ was used.

\section{Preprocessing}

For the purpose of data processing, we made use of the MATLAB programming environment (The MathWorks, Inc.), supplemented with processing routines from the SPM8b software package (Wellcome Department of Imaging Neuroscience, www.fil.ion.ucl.ac.uk/spm/) and the Group ICA of fMRI Toolbox (GIFT) v.1.3e (http://icatb.sourceforge .net/groupica.htm).

The functional imaging volumes were corrected for motion effects using 3D rigid body transformations. The anatomical images were coregistered to the functional volumes, and all images were normalized into Montreal Neurological Institute (MNI) stereotaxic space using affine transformations. Images were resampled at a 3-mm isotropic resolution and cropped in the anterior-to-posterior direction to a subvolume that comprised the acquired functional data $(-45 \leq y \leq+45)$. To improve signal-to-noise ratio characteristics, spatial smoothing was performed on the functional volumes using an isotropic 5-mm full width at half maximum Gaussian kernel. 
Finally, a logarithmic transformation was carried out in order to express all derived signal measures in units of percentage signal change.

\section{Multilinear regression analyses}

Two analyses were conducted by means of a general linear model (Friston et al., 2007). The most conventional one modeled signals of interest as a boxcar and, operationally, tested for greater fMRI signal during sound-on periods when compared with sound-off periods. The other one modeled signals of interest as a combination of sinusoidal functions, similar to a Fourier decomposition. In both cases, the periodicity of the modeled signal matched the fundamental presentation rate of the sound paradigm (i.e., 4 on/off stimulus blocks per run, or equivalently 9 acquisitions per on/off stimulus block, or 15 $\mathrm{mHz}$ ). The latter model also included higher harmonics. Various confounds were accounted for by including additional regressors that modeled (i) baseline and drift effects (four vectors modeling a third-order polynomial for each functional run separately), (ii) residual motion effects (six vectors containing the translation and rotation parameters along the $x$-, $y$-, and $z$-direction), and (iii) $T_{1}$-relaxation effects (two vectors containing the variable TRs, as well as their squares). For the resting-state data, the stimulus-related regressors were omitted from the model, but all mentioned confounds were included. After the models were evaluated for each individual subject, the regression coefficients of the signals of interest (i.e., the stimulus-related boxcar and sinusoidal functions) were entered into a mixed-effects group model and tested by means of ANOVA analyses (Ardekani and Kanno, 1998).

\section{Independent component analyses}

The confounds that were estimated in the regression model were subtracted from the preprocessed functional acquisitions. The resulting image volumes were then entered into an sICA. sICA unmixes signals into maximally independent sources that-in practice-result from minimally overlapping brain networks (Calhoun et al., 2001b; Hyvärinen and Oja, 2000; Langers, 2009). This technique differs from conventional regression analysis in a complete lack of prior assumptions about the temporal dynamics of evoked responses (except that responses in distinct networks are assumed not to be identical in order to be able to distinguish them).

The task/stimulus-driven data and the resting-state data were separately analyzed as follows. Per subject, all available data were reduced to 30 principal components, concatenated in time to form a single group-level dataset, and again reduced to 30 components (Calhoun et al., 2001a). Maximally independent components were extracted using the InfoMax algorithm (Bell and Sejnowski, 1995). The components' time courses were reconstructed in their original full length, obtaining concatenated signals spanning all subjects and all runs. The components were scaled such that their spatial maps and time courses had unit root-mean-square amplitude. The components that were obtained separately from the task/stimulus-driven and resting-state runs were reordered and retrospectively brought into one-to-one correspondence by maximizing the sum of the absolute inner products between matched component maps using the Kuhn-Munkres algorithm (Langers, 2010).
The time courses were then partitioned according to subject and run. For every subject, each component's mean time course over a single run was calculated. These mean time courses were Fourier transformed to determine their (complex-valued) spectra, and the group-average spectrum was computed. The components were ranked according to their absolute spectral power at a rate of four cycles per run, relative to the total signal power (Moritz et al., 2003). On the basis of their significance, the five components with the highest relative power were analyzed further. These were labeled as (I) the auditory system, including the inferior colliculi, medial geniculate bodies, and auditory cortex (inclusive of both primary cortex overlapping Heschl's gyrus and surrounding nonprimary areas); (II) the cingulo-insular network, including frontoinsular and anterior cingulate cortex; (III) the mediotemporal limbic lobe, including hippocampus and amygdala, but also the medial geniculate body; (IV) the basal ganglia, including striatum (caudate and putamen) and pallidum, but also parts of the thalamus, and partially overlapping with the mediotemporal limbic lobe; and (V) the posterior orbitofrontal cortex, also including superior medial frontal activity.

\section{(Partial) correlation analyses}

Various regions of interest (ROIs) were positioned on or near local maxima in the maps of the five independent components of interest. Each ROI consisted of two clusters of 19 voxels ( 1 central voxel plus its 6 face- and 12 edge-neighbors). Coordinates are included in Figure 4. The ROIs comprised, in component I, the [1] cochlear nuclei (CNU), [2] inferior colliculi (ICC), [3] medial geniculate bodies (MGB), and [4] primary auditory cortices (AUD); in component II, the [5] frontoinsular (INS) and [6] anterior cingulate (ACC) cortices; in component III, the [7] caudate nucleus (CAU); in component IV, the [8] amygdala (AMY) and [9] (para)hippocampus (HIP); and in component $\mathrm{V}$, the [10] posterior orbitofrontal cortex (OFC). The clusters for all but one ROI were positioned symmetrically in the left and right hemispheres; the clusters for the ACC were both centered on the midsagittal plane. Out of these 10 ROIs, the CNU was the only one that did not exceed threshold in the independent component map; it was nevertheless included because it appeared as a pair of coherent clusters in component I at more lenient thresholds, and it is an important auditory nucleus that has been reported in past fMRI studies (Hesselmann et al., 2001; Langers et al., 2005; Sigalovsky and Melcher, 2006; Yetkin et al., 2004). A superomedial cluster in component $\mathrm{V}$ was rejected from consideration, because it was located in a region with strong foldover artifacts.

For each of the 10 ROIs, a mean time course was determined by averaging the signals from the constituent voxels. In contrast to the preceding spectral analyses, these time courses were not averaged across subjects and runs, but consisted of a concatenation of the signals derived from all runs of all subjects. Functional connectivity maps were derived by calculating the Pearson correlation coefficients between an ROI's mean signal and the signals of each voxel in the brain (Horwitz, 2003; Lowe et al., 2000). In addition, partial correlation coefficients were determined between all pairs of ROIs, resulting in a connectivity measure that is similar to a path coefficient as derived from a structural equation model 
(Marrelec et al., 2006; Marrelec and Benali, 2009; Salvador et al., 2005).

\section{Statistics}

In the general linear regression model, the boxcar contrast was evaluated by means of a $t$-test, and the harmonic regressors were jointly assessed by means of an F-test (Ardekani and Kanno, 1998; Friston et al., 1995; Worsley and Friston, 1995). The derived statistical parametric maps were thresholded using regional false discovery rate criteria (Langers et al., 2007).

The significance of the spectral peak of the independent component time courses at 4 cycles per run was assessed by means of a Fourier F-test (Ardekani and Kanno, 1998; Purdon and Weisskoff, 1998). Similarly, the significance across subjects of the complex amplitude at four cycles per run was assessed by means of $F$-test statistics. In the connectivity model, the significance of all 45 possible connections between ROIs was evaluated by means of a bootstrap resampling method. Concatenated time courses were repeatedly constructed by random sampling from the available runs (with replacement), and partial correlation coefficients were determined on the basis of the resampled data. Resampling within runs was avoided, because that would destroy the temporal characteristics of the signal (e.g., autocorrelations, spectrum, and remaining scanner drift). One million such outcomes were used to construct probability distributions for the connectivity levels of all pairwise connections between ROIs, and these were used to assess the significance of deviations from zero. A Bonferroni correction was applied for the total number of connections in the model.

\section{Results}

\section{Multilinear regression analyses}

Figure 1a shows the results of the conventional analysis that contrasted the fMRI signal between the sound-on and -off periods. This analysis assumes that any increase in activity (seen as an increase in image signal) occurred immediately following each sound presentation, except for a small hemodynamic delay. Group-level activation was revealed in various centers of the auditory pathway: the inferior colliculi in the midbrain, the medial geniculate bodies in the thalamus, and wide expanses of the superior temporal lobes corresponding to both primary and nonprimary auditory cortical areas. Little or no activation was observed outside these brain regions.

\section{Independent component analyses}

Complementarily, an analysis uninformed by the stimulus was carried out. Specifically, an sICA decomposed the data into 30 components, each consisting of a spatial map of component amplitude and a corresponding time course quantifying the variation over time. In addition to components with a neural basis, components reflecting artifacts, such as those associated with subject movement or the variable TR of the MRI sequence, were also extracted, as is commonly the case with sICA. Fourteen components were classified as artifactual, because they were largely confined to the "edges" of the brain, were spread out widely but sparsely, or tended to colocalize with CSF compartments (Langers, 2010). The remaining 16 a

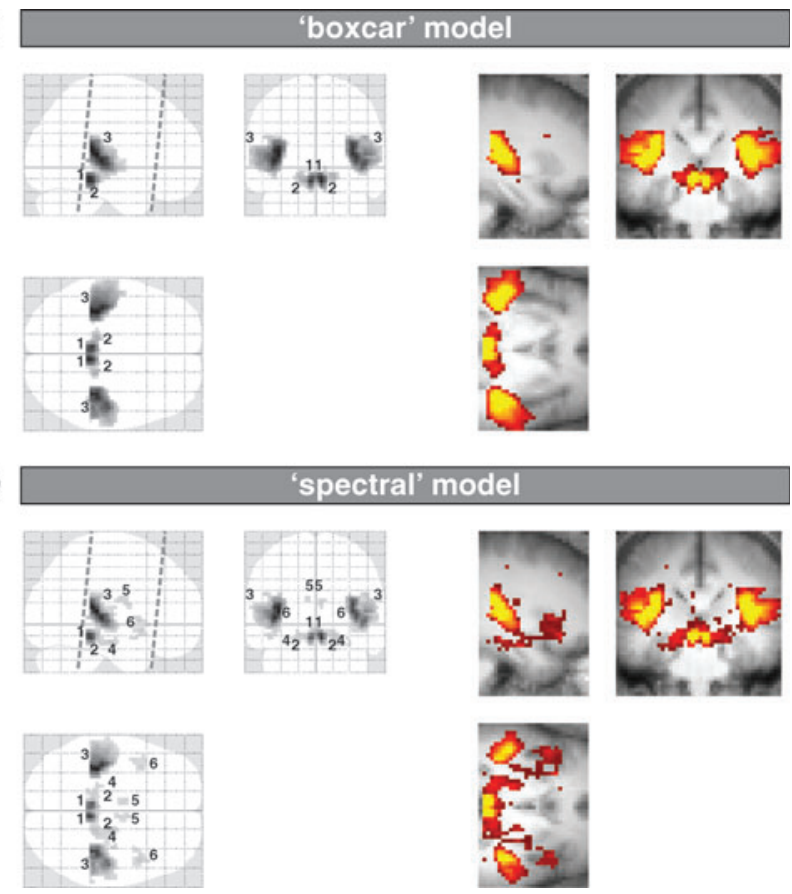

FIG. 1. (a) Conventional regression analysis based on a phase-sensitive "boxcar" model to detect stimulus-synchronized responses mainly revealed activation in classical auditory structures, like [1] the inferior colliculi of the midbrain, [2] the medial geniculate bodies of the thalamus, and [3] the auditory cerebral cortices. (b) A more general analysis based on a phase-insensitive "spectral" model to detect any responses with the same periodicity as the sound stimuli additionally revealed activation in nonauditory structures such as [4] the hippocampus, [5] the caudate nucleus, and [6] the insula. In the left panels, stimulus-related brain activation was overlaid on a "glass brain" ( $p<0.001$ uncorrected; minimum cluster size 10 voxels); the right panels show cross-sectional anatomical images with all suprathreshold voxels overlaid by means of a color-coded maximum intensity projection $(q<0.05$ regional false discovery rate).

components were tentatively classified as neural on the basis of their coherent spatial distribution that well corresponded with particular gray matter brain structures or with networks identified by others (Allen et al., 2011; Schöpf et al., 2010; Smith et al., 2009). These 16 components were assessed for influences of sound stimulation by calculating the percentage of total power in the time course that occurred at the rate of the sound presentation cycles and by ranking them accordingly (Fig. 2). The relative power was statistically significant for four components, which were subsequently analyzed in more detail (I-IV; $p<0.01$ ). A fifth component (V) that bordered on significance was also considered, as its power was closer to that of components II-IV than to the components with the next lower relative power.

The component showing the greatest relative power at the rate of sound presentation was confined to the classical auditory pathway (Fig. 3a, component I). It comprised auditory cortical areas in the superior temporal plane, the medial geniculate bodies, and the inferior colliculi. These structures are the same as those showing activation according to the conventional boxcar analysis of Figure 1a. Accordingly, the 


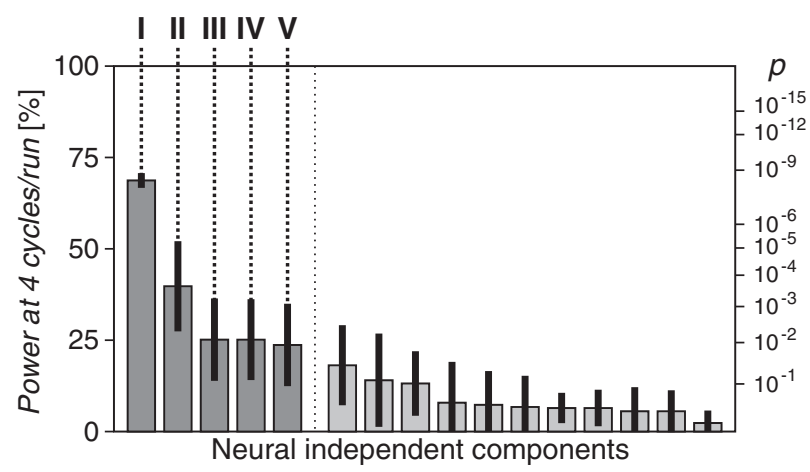

FIG. 2. Five independent components showed soundrelated responses (dark bars; labeled I-V). These were distinguished from the remaining independent components of neural origin (light bars) as follows. First, for each of the 16 independent components, the power at the stimulus presentation rate (four cycles/run) was calculated, expressed as a percentage of the total signal power (left axis), and assessed for significance according to a Fourier F-test (right axis). Power was significant $(p<0.01)$ for components I-IV and nearly so for component $\mathrm{V}$. The error bars in the figure indicate standard errors of the outcome as calculated by bootstrap resampling across subjects.

time course of this auditory component faithfully followed the on/off cycles of the sound stimulus (Fig. 3b), even though the sICA was not informed by the timing of the stimulus.

The other four components included, almost exclusively, structures outside the classical auditory system (Fig. 3a, components II-V). The time courses of these components showed quasiperiodic changes related to the sound stimulus presentations (Fig. 3b), but not the same faithful following of the stimulus on/off periods as the auditory component. This different behavior can be quantitatively seen in Figure 3c, which shows polar plots of the phase and amplitude of a sinusoidal fit to each component's time course for each subject. The average phases of the nonauditory components are shifted forward by approximately $110-160^{\circ}$ (1.9-2.8 radians) relative to that of the auditory component. The phases of the nonauditory components also show more variability across subjects than does the phase of the auditory component. Still, the complex amplitude was significantly different from zero at the group level $(p<0.05)$ in all components except the mediotemporal limbic one (III), which showed a trend $(p=0.1)$.

To investigate whether the lack of activation in nonauditory centers according to conventional boxcar analysis in Figure 1 a could be attributed to the observed phase differences between classical auditory and nonauditory structures, the analysis was extended to capture any response with the same periodicity as the sound cycles regardless of phase (Fig. 1b). In addition to revealing activation in auditory centers, the latter analysis also revealed significant activation in areas outside the classical auditory system, including the hippocampus, insula, caudate nucleus, and superior thalamus, most of which were included in one of the independent components II-V. Thus, differences in the phase of response across structures at least partially explained why the nonauditory areas of components II-V were not detected by conventional methodology.

\section{Partial correlation analyses}

A functional connectivity analysis was performed to confirm the correlations between brain areas implied by the sICA and to study possible associations between the identified components. Ten ROIs were defined, coinciding with local maxima in the five independent component maps (I-V). For each ROI, coefficients that quantified the temporal correlation with each voxel in the brain were determined. Figure 4a shows the resulting maps of functional connectivity.

From the CNU, protrusions of functional connectivity were seen to ascend along the tracts of the lateral lemnisci toward the ICC. The ICC primarily showed connectivity with superior temporal areas of cerebral cortex. In stark contrast to $\mathrm{CNU}$ and ICC, the MGB showed high levels of functional connectivity with many brain regions: in addition to the superior temporal lobe, these included mediotemporal areas, superior thalamus and basal ganglia, and cingulate cortex. In comparison, the connectivity of AUD was much more limited, most notably including superior thalamus and insula. With regard to the nonauditory ROIs, the INS, ACC, and CAU appeared to be tightly interconnected, as were AMY and HIP.

The time courses of the aforementioned ROIs (Fig. 4b) once more showed that the responses in the four auditory ROIs (CNU, ICC, MGB, and AUD) were closely synchronized with the stimulus presentations. The other ROIs displayed more or less periodic signal oscillations as well, but showed a systematic phase shift relative to the auditory ROIs, as illustrated in the polar plots of Figure 4c. With the exception of only AMY and CAU, all ROIs showed significantly nonzero (complex) amplitudes at the group level $(p<0.05)$.

To better understand the interactions between the 10 ROIs, a partial correlation analysis was carried out. Outcomes reflect the strength of pairwise correlations between ROIs that cannot be explained by mutual correlations with other ROIs. As such, partial correlations correct for contributions caused by shared input or mediated connections. Results of this analysis are shown in Figure 5a, where only the most significant correlations ( $p<0.001$, corrected) are indicated. The shading groups ROIs by the independent components (I-V) in which they reached suprathreshold $z$-scores $(z>2.5)$.

ROIs belonging to the same independent component typically showed high partial correlations, indicating strong direct connectivity. Almost all ROIs showed connectivity with an auditory ROI; the single exception was OFC, which showed connectivity with INS and HIP, but not with any of the auditory processing centers. Two ROIs, MGB and INS, showed particularly extensive connectivity with other areas. The MGB showed connectivity with ICC and AUD and with multiple nonauditory structures, like CAU, HIP, and AMY. Interestingly, the MGB's partial correlation with HIP was just as great as with ICC and greater than with AUD. The second nexus, INS, showed significant correlations with AUD, ACC, CAU, HIP, and AMY.

\section{Resting state}

Because the partial correlations between ROIs yielded by the connectivity analysis exclude mutual correlations with other ROIs, it should also largely exclude correlations attributable to a shared drive such as the sound stimulus. This raises the interesting possibility that the effective connectivity 


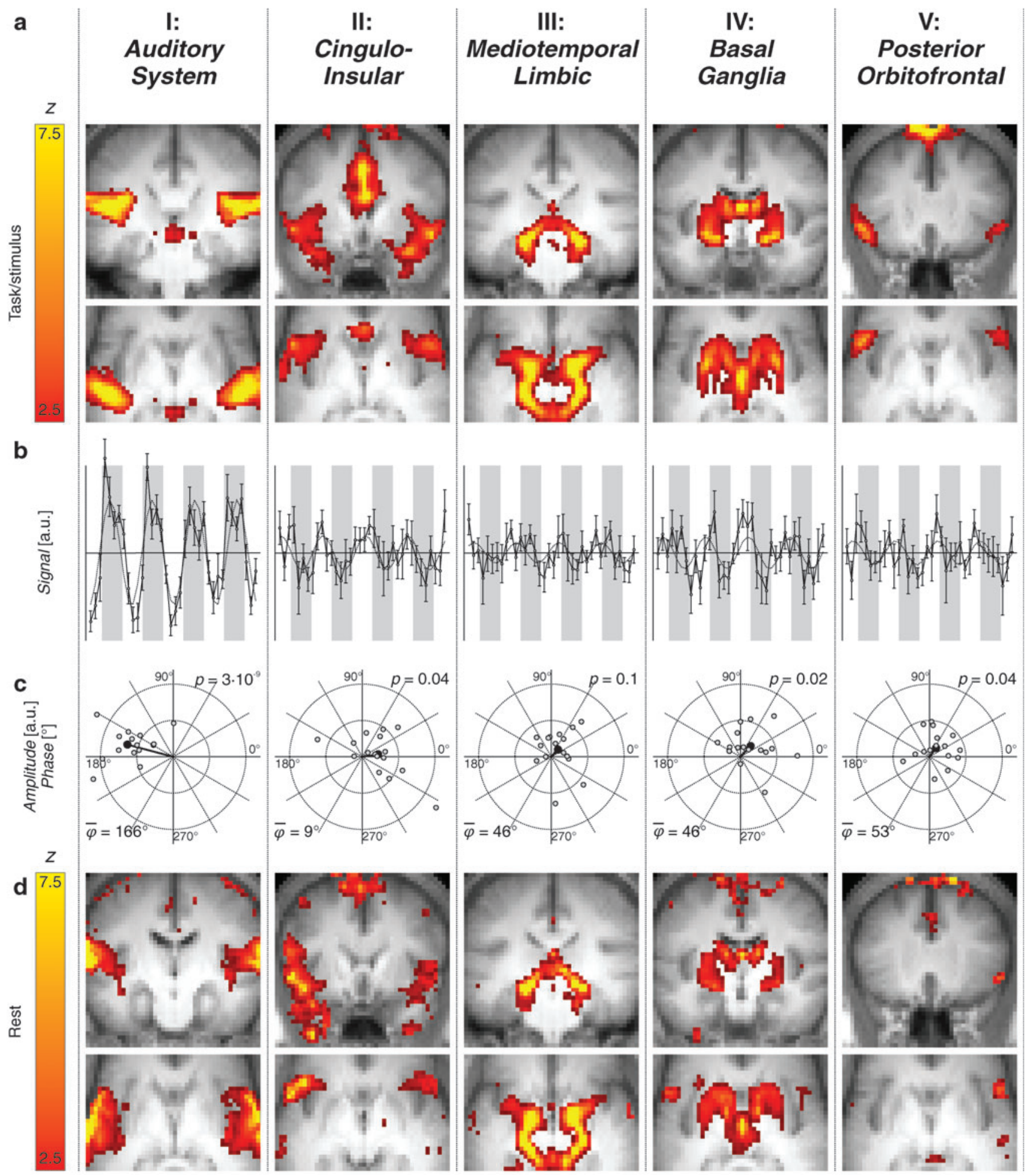

FIG. 3. Five independent components (I-V) showing sound-related responses (a-c) are also present at rest (d). (a) The maps show the spatial distribution of component amplitude, scaled to unit variance and thresholded at $z=2.5$. (b) Corresponding time courses were averaged across subjects and runs; error bars indicate the standard error across subjects. (c) The complex amplitude of the spectrum at four cycles/run was plotted in polar form. Each gray dot of a given panel represents an individual subject; the group mean is shown in black. Mean phase angles $\varphi$ and group-level $p$-values according to an ANOVA are reported. (d) Five independent components that closely matched those of components I-V in panel a were independently extracted from the resting-state runs. 


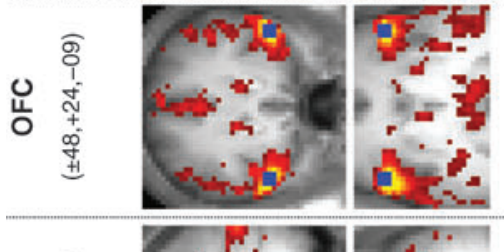

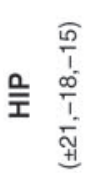
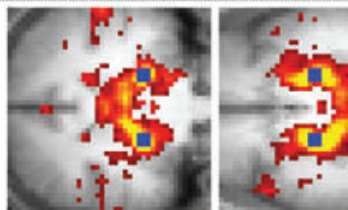

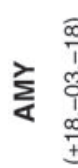

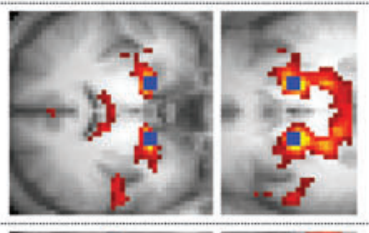

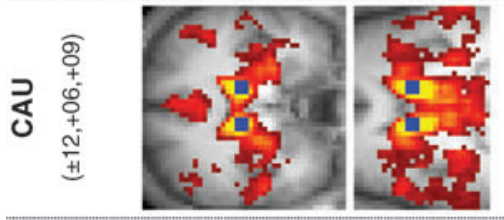
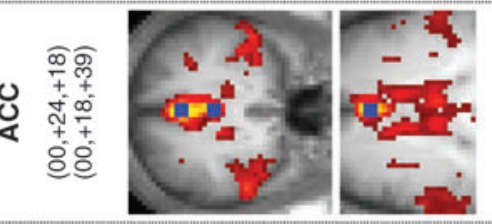

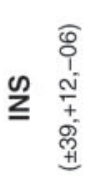
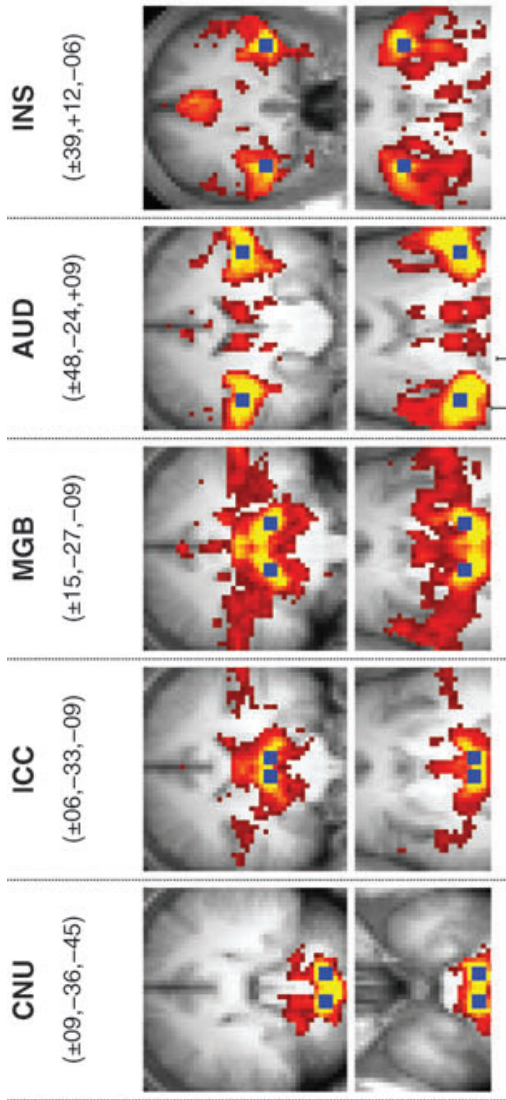

ซ

$$
\llbracket \underset{\infty}{\infty}
$$

sn!nu!̣smse
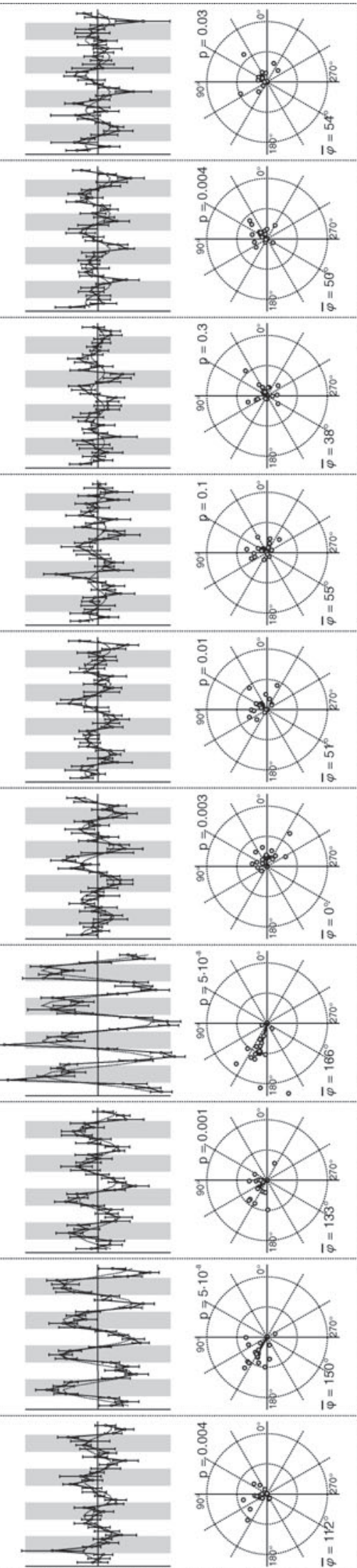

[n'r] |вu6!!
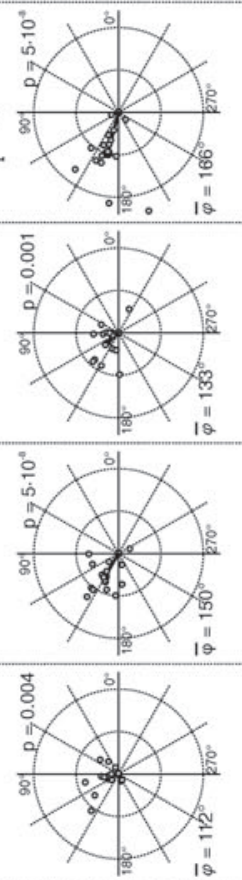
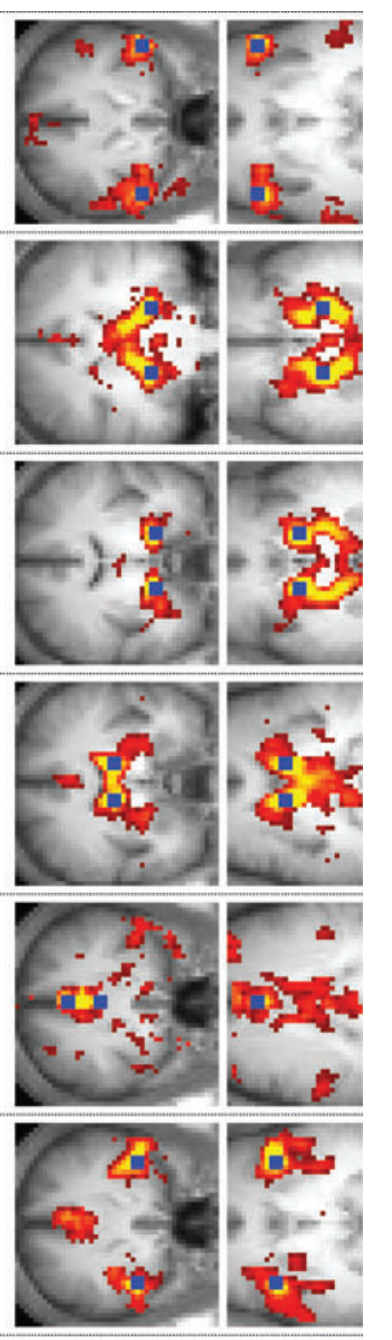

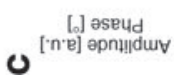
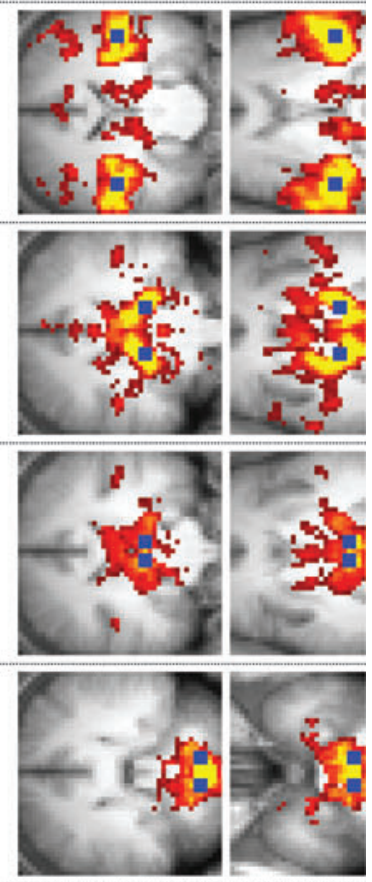

똥

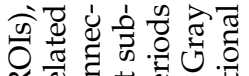

踏

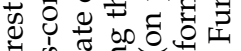

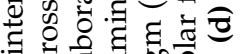

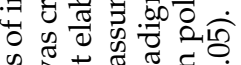

3 30 .

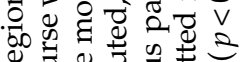

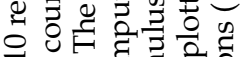

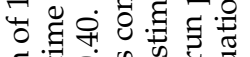

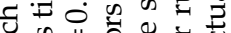

ฐँ

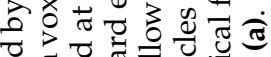

尊

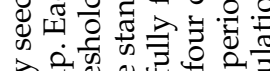

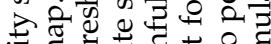

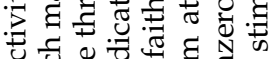

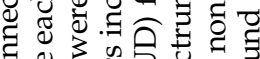

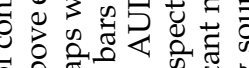

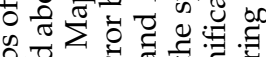

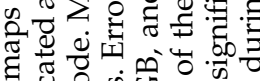

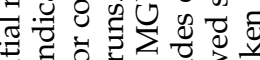

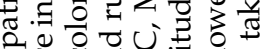

की 0 远

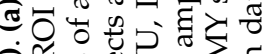

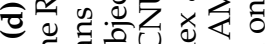

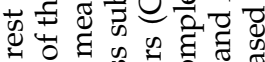

过

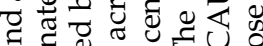

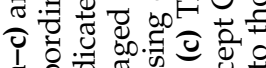

@ 0.0

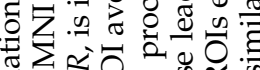

क

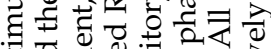

क

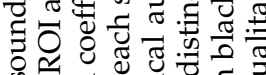

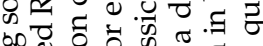

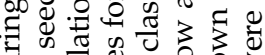

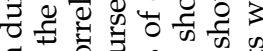

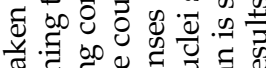

눙

त्ञ

준 웅

象

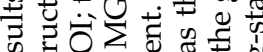

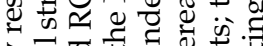

可议

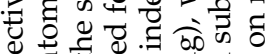

द्व

80

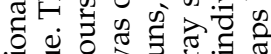

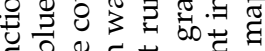

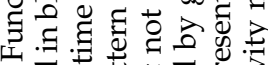

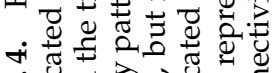

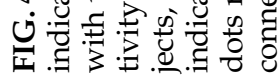


a

Task/Stimulus

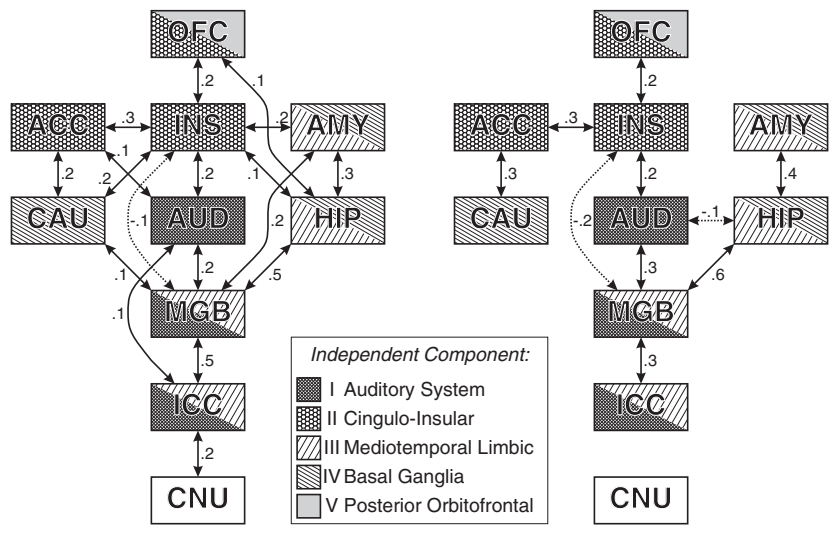

FIG. 5. Effective connectivity between auditory and nonauditory ROIs. The ROIs are the same as in Figure 4 and correspond to local maxima in the spatial maps of independent components I-V. Shading groups the ROIs according to the independent components in which they reached suprathreshold $z$-scores ( $z>2.5$; see Fig. 3a). The connectivities shown for (a) the task- and stimulus-driven condition and (b) the resting-state condition are based on separate analyses. Only highly significant connections $(p<0.001$, corrected for multiple comparisons) are indicated (lines with arrows, labeled with the corresponding partial correlation coefficients; solid and dotted lines indicate positive and negative correlations, respectively). Significance was assessed by means of a bootstrap resampling technique. Many of the connections apparent during the sound-stimulated condition were also apparent during the resting state. For both datasets the MGB and INS were nexes of connectivity in that they showed significant effective connectivity with the greatest number of other ROIs.

pattern apparent in sound-stimulated subjects might also be present in unstimulated subjects. To test this, additional measurements were performed in the absence of an explicit sound stimulus, that is, during "rest".

An sICA of the resting-state data revealed that the five sound-responsive independent components identified in sound-stimulated subjects could also be extracted in the absence of sound stimulation (Fig. 3d), perhaps with the exception of the posterior orbitofrontal component $(\mathrm{V})$. The spatial maps of corresponding independent components were highly similar, especially those for components I, III, and IV (compare Fig. 3d with 3a). The time courses during rest (not shown) were essentially random because of the lack of an invariable stimulus/task paradigm.

Functional connectivity maps revealed more or less the same relationships between structures as seen during sound stimulation, although the observed connectivity levels were slightly lower overall (compare Fig. 4d with 4a). Concurrently, the resulting partial correlations (Fig. 5b) were generally less significant than in the sound-stimulated case, but key observations remained, such as: the connectivities within an independent component were typically significant; the MGB and INS remained hubs of connectivity; the MGB showed stronger connectivity with the HIP than with the AUD; and OFC did not directly connect to any classical auditory processing center.

\section{Discussion}

Our results demonstrate sound-related activity in widespread areas outside the auditory pathway as well as within it. In particular, cingulo-insular cortex, mediotemporal limbic lobe, basal ganglia, and posterior orbitofrontal cortex showed significant modulations of activity at the rate of the sound stimulation cycles, despite subjects' passivity with respect to the auditory domain. The only explicit task, a visual one requiring little effort, was uncorrelated with the sound presentations and cannot account for the detected variations in activity at the sound on/off rate. Although power at this rate was less in nominally "nonauditory" centers compared with classical auditory ones, it was nevertheless statistically significant and apparent by eye in group-averaged signal time courses. This finding in nonauditory centers is unlikely to represent the activity of a few isolated neurons. Rather, it indicates acoustically driven changes in activity in entire neuronal populations.

Sound-related changes in the activity of nonauditory centers were revealed by sICA, which also separated the centers into multiple independent components, or "networks" of brain sites bound by coordinated activity. This binding was confirmed by the connectivity analyses in that the greatest partial temporal correlations occurred between structures residing in the same network. An important point is that the sound stimulus was not required for the binding between structures, as demonstrated by the fact that an sICA of data taken in subjects at rest still revealed components I-IV (and, questionably, V), and the greatest partial correlations were still between structures within the same network. Although scanner-generated acoustic background noise has the potential for inducing temporal correlations between brain sites even during the acquisition of resting data, functional brain connectivity measures have been shown to be relatively insensitive to the presence of acoustic scanner noise (Langers and van Dijk, 2011), and the employed sparse clustered imaging sequence further mitigated against this (Edmister et al., 1999; Hall et al., 1999). Thus, we propose that (1) components I-IV reflect "intrinsic" networks, meaning networks reliant on endogenous rather than sound-evoked activity fluctuations for their formation; and [2] upon presentation of a sound stimulus, these already-existent, intrinsic networks are recruited en toto into engagement by sound.

The connectivity revealed by the sICA and effective connectivity analysis are entirely plausible from an anatomical standpoint; there is evidence in mammalian species for direct anatomical connections between most of the interconnected ROIs in Figure 5 (Augustine, 1996; Bamiou et al., 2003; Budinger et al., 2008; Cavada et al., 2000; Clugnet et al., 1990; Doron and Ledoux, 1999; Eblen and Graybiel, 1995; LeDoux et al., 1990; Rosell and Giménez-Amaya, 1999; Winer and Lee, 2007). That said, it is important to recognize that the connectivities established here based on functional data are not equivalent to anatomical ones, and the direct anatomical projections reported in the literature may not have a one-to-one correspondence to the connectivities in Figure 5.

In addition to the correlated activity among centers within a given independent component, there were correlations between centers residing in different components. Two centers were particularly involved in such intercomponent correlations: MGB and INS. Because these are heterogeneous 
centers, both functionally and anatomically, it is possible that different subdivisions are responsible for the connectivities with the other considered brain regions. The resolution of our imaging does not allow us to distinguish between this possibility and a completely opposing one in which the connectivities are mediated by intermingled neuronal types, or even the same neurons. In any case, the results clearly bolster proposals that anterior insula is a nexus of connectivity (Augustine, 1996; Craig, 2009; Menon and Uddin, 2010), and that the same may be true for the thalamic MGB.

Although five networks (sICA components I-V) showed sound-related variations in activity, the meaning of these variations likely differs between networks.

Interpretation of the auditory component (I) is straightforward; cortical and subcortical neuronal populations encoding the sound stimulus increased/decreased their firing rate each time the sound was turned on/off. The detailed temporal dynamics differ somewhat between the various subdivisions of the auditory system. For instance, sustained responses are commonly observed in subcortical nuclei, whereas onset and offset responses start to gain importance at higher cortical levels. Our results are in general agreement with results that were previously reported in more detail (Sigalovsky and Melcher, 2006). At various hierarchical levels in the auditory pathway, spectrotemporal acoustic features are transformed and re-encoded into more abstract representations of sound objects (Griffiths and Warren, 2004; Harms et al., 2005; Harms and Melcher, 2002; Husain et al., 2004; Leaver and Rauschecker, 2010; Nelken, 2004; Zatorre et al., 2004). Such representations likely contain information related to auditory object recognition and the nature and location of the sound source that are conveyed through ventral and dorsal "what" and "where" streams in a form that can be easily integrated with similar information arising from other sensory modalities (Mesulam, 1998; Rauschecker and Tian, 2000). Our connectivity results suggest the insula as a possible target of such streams.

Component II, comprising frontoinsular and anterior cingulate cortex, bears a striking resemblance to the "salience network" identified by Seeley et al. (2007). The frontoinsular cortex in particular has been implicated in bottom-up detection of salient events and in coordinating appropriate responses by switching between large-scale networks to facilitate access to attention and working memory resources (Medford and Critchley, 2010; Menon and Uddin, 2010). Activity in this network has been shown to be correlated with improved detection thresholds for auditory stimuli in particular, substantiating its role in the direction of attentional resources toward the auditory domain (Sadaghiani et al., 2009). Affiliation of component II with a salience network suggests a possible account for the phase-shifted time course of this component compared with the auditory one. During the sound-on periods, the task-related stimuli compete with the distracting noise stimuli. As a result, the behaviorally relevant visual input may achieve lower salience. In contrast, during sound-off periods, the visual stimuli are unchallenged and more salient. If activation of component II reflects task-related stimulus salience, it should therefore be in opposite phase to the sound presentations. The phase difference indeed approached $180^{\circ}$. An alternative interpretation of component II is that it relates to the default mode network (DMN). The DMN comprises structures proposed to mediate introspection or self-referential thought and shows reduced activity during a directed task (Fox and Raichle, 2007; Greicius et al., 2003; Raichle and Snyder, 2007). This deactivation has been primarily documented during active tasks, but it has also been reported during passive sensation of stimuli (Greicius and Menon, 2004) and would thus be consistent with a $\sim 180^{\circ}$ phase difference.

Component III includes the hippocampus and amygdala in the mediotemporal limbic lobe, centers involved in declarative memory, fear conditioning, and mediation of affect (Phelps and LeDoux, 2005). Interestingly, this component overlapped with the auditory component (I) in the MGB, which may relate to the suggested role of posterior (para)hippocampal areas in the control of thalamic auditory gating (Boutros et al., 2008). The time course of this component was shifted forward by approximately $120^{\circ}$ relative to the auditory response, indicating an increase in signal prior to the onset of sound stimulation. There are at least two possible interpretations for such a phase shift. One is that it reflects anticipation of the sound stimulus, which was presented in a regular on/off fashion and thus occurred predictably (Leaver et al., 2009). This explanation would be consistent with the slightly more irregular appearance of the time course of component III around the first sound presentation. Another possible explanation is related to habituation (Mutschler et al., 2010). Although the sound stimuli were irrelevant for the task, they may have captured some of the subject's attention. Short-term habituation to the persisting noise may lead to a decline of the sound-evoked response already during the presentation period itself. Both of these effects, anticipation (before stimulus onset) and habituation (after stimulus onset), may work together to shift the oscillation of instantaneous response amplitude forward in time.

Component IV, while spreading into the hippocampus and amygdala, was maximal in amplitude in the basal ganglia, well known for their role in procedural memory, reward, learning, motor planning, and goal-directed behavior (Graybiel, 2005; Poldrack et al., 2001). Like component III, component IV showed a time course phase shift of approximately $120^{\circ}$, which may again be related to anticipation and/or habituation. Despite the similarity in sound-related activity modulations, these two components were distinguished from one another by the sICA, presumably because of differences in variations in intrinsic activity.

Finally, component $\mathrm{V}$ was weakest of all components considered, with its sound responsiveness only bordering on significance. Posterior OFC is known to receive input from all sensory modalities, including audition (Kringelbach and Rolls, 2004). It is hypothesized to be involved in the processing of reward and project to the striatum in the basal ganglia (IV). However, activity in OFC was only indirectly related to that in auditory areas according to our connectivity analyses and did not show direct connectivity with the basal ganglia (represented through CAU) either. Possibly, component V coincides with a central executive control network that includes OFC (Mazoyer et al., 2001; Seeley et al., 2007). The fact that this control network is modulated by frontoinsular cortex (Medford and Critchley, 2010; Sridharan et al., 2008) combined with our observation of connectivity between INS and OFC lend support to this interpretation.

An interesting aspect of the data is the substantial intersubject variability in the phase of components II-V, which 
contrasted with the much more consistent phases in component I comprising classical auditory centers. Some of this variability may reflect the fact that sound-induced fluctuations in image signal were lower in relation to noise for components II-V. However, the variability may also reflect true intersubject variability in the timing of interactions between sound-related changes in activity and neuronal activity associated with the myriad of functions in which the nonauditory centers comprising components II-V are known to be engaged. Such variability is to be expected in a near-passive experimental design like that of the present study.

It is important to recognize that the present study has not necessarily identified all nonauditory areas with soundevoked activity changes for at least two reasons. First, there is always the possibility that sound-responsive neurons are too low in density in a given region to yield a significant response in fMRI. In other words, components that were not analyzed in the present study because of a low relative power at the sound presentation rate (Fig. 2) may nevertheless have included some sound-related activity. Second, although assumptions about the nature of any sound-related activation were relaxed compared with conventional analyses, they were not entirely removed because of the way that sound-related sICA components were identified. The sICA made no assumptions about the relationship between sound stimulus and brain activation, but the percentage power calculation, which was used to single out components I-V, did. Signal variations of a component must be sufficiently related to the sound cycling for the relative power to be high. Thus, although the present study indicates sound-related activity variations in certain nonauditory brain areas, it cannot rule out the possibility of sound-related activity in still others.

Another obvious limitation of our study lies in the partial volume acquisitions that did not include the most anterior and posterior regions of the brain. This choice was motivated by the requirements to use short acquisition sequences to avoid excessive scanner noise in combination with sufficient spatial in-plane resolution to distinguish small subcortical auditory nuclei (Kovacs et al., 2006; Sigalovsky and Melcher, 2006; Talavage et al., 1999). Moreover, our initial goal was to reveal interactions that subserve auditory gating, which were primarily expected to occur in the limbic system and diencephalon (Rauschecker et al., 2010). As a result, we are currently unable to fully characterize the activity and interactions in the frontal and occipital lobes, including regions related to the processing of, e.g., vision, attention, and introspection (Leaver et al., 2009; Romanski et al., 1999). The series of intrinsic networks reported in Figure 3 should therefore not be considered exhaustive, and the derived network graph in Figure 5 is likely incomplete. Nevertheless, even with a limited field of view, we were able to newly reveal involvement of "nonauditory" regions in the processing of basic task-irrelevant sound stimuli.

Although the present data demonstrate sound-induced changes in population neural activity outside the classical auditory system, they do not prove a functional significance of these responses. One possibility is that the identified acoustic responses of nonauditory areas were merely an epiphenomenon arising from the fact that these areas need to register behaviorally relevant input in other contexts. On the other hand, it might be more reasonably argued that all observed sound-evoked responses have functional significance, in which case the nonauditory areas recruited by sound may influence how the sound itself is processed and/or consciously perceived. Recent data showing reduced functional connectivity between putamen and auditory cortex during loss of consciousness provides direct evidence in support of this interpretation for component III (Mhuircheartaigh et al., 2010). Further, dysfunction in the coordination of the activity in precisely these networks may underlie disorders such as tinnitus and hyperacusis (Rauschecker et al., 2010) and possibly plays a role in, for example, dyslexia, autism and schizophrenia (Ahissar, 2007; Feinberg and Guazzelli, 1999; Nieto Del Rincón, 2008; Uddin and Menon, 2009; Williamson, 2007).

In summary, when all of our data are considered collectively, the picture is one of multiple networks of coordinated activity that are responsive to sound. The networks are intrinsic in that they are present in the absence of any explicit stimulation. All but one network comprise, almost exclusively, structures outside the classical auditory system. Soundrelated activity in these nonauditory brain centers may only be detected if the assumption that evoked activation faithfully follows stimulation is relaxed.

\section{Acknowledgments}

The authors acknowledge the generous contributions of Jianwen $\mathrm{Gu}$, who performed the audiological assessments, constructed the stimulus paradigm, and participated in the fMRI acquisitions, and Christine Hsieh, who helped characterize the influence of various MRI artifacts in the sICA analyses. Financial support was provided by the Tinnitus Research Initiative, Kenneth Griffin, Jack and Shelley Blais, and NIH (P30DC005209). Partial support was also provided by the National Center for Research Resources (P41RR14075) and the Mental Illness and Neuroscience Discovery (MIND) Institute. The author D.R.M.L. was partly funded by VENI research grant (016.096.011) from The Netherlands Organization for Scientific Research (NWO) and The Netherlands Organization for Health Research and Development (ZonMw).

\section{Author Disclosure Statement}

No competing financial interests exist.

\section{References}

Ahissar M. 2007. Dyslexia and the anchoring-deficit hypothesis. Trends Cogn Sci (Regul. Ed.) 11:458-465.

Allen EA, Erhardt EB, Damaraju E, Gruner W, Segall JM, Silva RF, Havlicek M, Rachakonda S, Fries J, Kalyanam R, et al. 2011. A baseline for the multivariate comparison of restingstate networks. Front Syst Neurosci 5:2.

Ardekani BA, Kanno I. 1998. Statistical methods for detecting activated regions in functional MRI of the brain. Magn Reson Imaging 16:1217-1225.

Augustine JR. 1996. Circuitry and functional aspects of the insular lobe in primates including humans. Brain Res Brain Res Rev 22:229-244.

Bamiou D-E, Musiek FE, Luxon LM. 2003. The insula (Island of Reil) and its role in auditory processing. Literature review. Brain Res Brain Res Rev 42:143-154.

Bell AJ, Sejnowski TJ. 1995. An information-maximization approach to blind separation and blind deconvolution. Neural Comput 7:1129-1159. 
Boutros NN, Mears R, Pflieger ME, Moxon KA, Ludowig E, Rosburg T. 2008. Sensory gating in the human hippocampal and rhinal regions: regional differences. Hippocampus 18:310316.

Boutros NN, Trautner P, Rosburg T, Korzyukov O, Grunwald T, Schaller C, Elger CE, Kurthen M. 2005. Sensory gating in the human hippocampal and rhinal regions. Clin Neurophysiol 116:1967-1974.

Budinger E, Laszcz A, Lison H, Scheich H, Ohl FW. 2008. Nonsensory cortical and subcortical connections of the primary auditory cortex in Mongolian gerbils: bottom-up and topdown processing of neuronal information via field AI. Brain Res 1220:2-32.

Calhoun VD, Adali T, Pearlson GD, Pekar JJ. 2001a. A method for making group inferences from functional MRI data using independent component analysis. Hum Brain Mapp 14:140151.

Calhoun VD, Adali T, Pearlson GD, Pekar JJ. 2001b. Spatial and temporal independent component analysis of functional MRI data containing a pair of task-related waveforms. Hum Brain Mapp 13:43-53.

Cavada C, Compañy T, Tejedor J, Cruz-Rizzolo RJ, ReinosoSuárez F. 2000. The anatomical connections of the macaque monkey orbitofrontal cortex. A review. Cereb Cortex 10: 220-242.

Clugnet MC, LeDoux JE, Morrison SF. 1990. Unit responses evoked in the amygdala and striatum by electrical stimulation of the medial geniculate body. J Neurosci 10:1055-1061.

Craig ADB. 2009. How do you feel-now? The anterior insula and human awareness. Nat Rev Neurosci 10:59-70.

Doron NN, Ledoux JE. 1999. Organization of projections to the lateral amygdala from auditory and visual areas of the thalamus in the rat. J Comp Neurol 412:383-409.

Eblen F, Graybiel AM. 1995. Highly restricted origin of prefrontal cortical inputs to striosomes in the macaque monkey. J Neurosci 15:5999-6013.

Edmister WB, Talavage TM, Ledden PJ, Weisskoff RM. 1999. Improved auditory cortex imaging using clustered volume acquisitions. Hum Brain Mapp 7:89-97.

Ehret G. 1997. The auditory cortex. J Comp Physiol A 181:547557.

Feinberg I, Guazzelli M. 1999. Schizophrenia-a disorder of the corollary discharge systems that integrate the motor systems of thought with the sensory systems of consciousness. Br J Psychiatry 174:196-204.

Fox MD, Raichle ME. 2007. Spontaneous fluctuations in brain activity observed with functional magnetic resonance imaging. Nat Rev Neurosci 8:700-711.

Friston KJ, Ashburner JT, Kiebel SJ, Nichols TE, Penny WD. 2007. Statistical Parametric Mapping: The Analysis of Functional Brain Images, 1st ed. Amsterdam, The Netherlands: Elsevier/Academic Press.

Friston KJ, Holmes AP, Poline JB, Grasby PJ, Williams SC, Frackowiak RS, Turner R. 1995. Analysis of fMRI time-series revisited. Neuroimage 2:45-53.

Graybiel AM. 2005. The basal ganglia: learning new tricks and loving it. Curr Opin Neurobiol 15:638-644.

Greicius MD, Krasnow B, Reiss AL, Menon V. 2003. Functional connectivity in the resting brain: a network analysis of the default mode hypothesis. Proc Natl Acad Sci U S A 100:253-258.

Greicius MD, Menon V. 2004. Default-mode activity during a passive sensory task: uncoupled from deactivation but impacting activation. J Cogn Neurosci 16:1484-1492.

Griffiths TD, Warren JD. 2004. What is an auditory object? Nat Rev Neurosci 5:887-892.
Gu JW, Halpin CF, Nam E-C, Levine RA, Melcher JR. 2010. Tinnitus, diminished sound-level tolerance, and elevated auditory activity in humans with clinically normal hearing sensitivity. J Neurophysiol 104:3361-3370.

Guimaraes AR, Melcher JR, Talavage TM, Baker JR, Ledden P, Rosen BR, Kiang NY, Fullerton BC, Weisskoff RM. 1998. Imaging subcortical auditory activity in humans. Hum Brain Mapp 6:33-41.

Hall DA, Haggard MP, Akeroyd MA, Palmer AR, Summerfield AQ, Elliott MR, Gurney EM, Bowtell RW. 1999. "Sparse" temporal sampling in auditory fMRI. Hum Brain Mapp 7:213-223.

Harms MP, Guinan JJ, Sigalovsky IS, Melcher JR. 2005. Shortterm sound temporal envelope characteristics determine multisecond time patterns of activity in human auditory cortex as shown by fMRI. J Neurophysiol 93:210-222.

Harms MP, Melcher JR. 2002. Sound repetition rate in the human auditory pathway: representations in the waveshape and amplitude of fMRI activation. J Neurophysiol 88:1433-1450.

Hesselmann V, Wedekind C, Kugel H, Schulte O, Krug B, Klug N, Lackner KJ. 2001. Functional magnetic resonance imaging of human pontine auditory pathway. Hear Res 158:160-164.

Horwitz B. 2003. The elusive concept of brain connectivity. Neuroimage 19:466-470.

Husain FT, Tagamets M-A, Fromm SJ, Braun AR, Horwitz B. 2004. Relating neuronal dynamics for auditory object processing to neuroimaging activity: a computational modeling and an fMRI study. Neuroimage 21:1701-1720.

Hyvärinen A, Oja E. 2000. Independent component analysis: algorithms and applications. Neural Netw 13:411-430.

Kovacs S, Peeters R, Smits M, De Ridder D, Van Hecke P, Sunaert S. 2006. Activation of cortical and subcortical auditory structures at $3 \mathrm{~T}$ by means of a functional magnetic resonance imaging paradigm suitable for clinical use. Invest Radiol 41:8796.

Kringelbach ML, Rolls ET. 2004. The functional neuroanatomy of the human orbitofrontal cortex: evidence from neuroimaging and neuropsychology. Prog Neurobiol 72:341-372.

Langers DRM. 2009. Blind source separation of fMRI data by means of factor analytic transformations. Neuroimage 47:77-87.

Langers DRM. 2010. Unbiased group-level statistical assessment of independent component maps by means of automated retrospective matching. Hum Brain Mapp 31:727-742.

Langers DRM, Jansen JFA, Backes WH. 2007. Enhanced signal detection in neuroimaging by means of regional control of the global false discovery rate. Neuroimage 38:43-56.

Langers DRM, van Dijk P. 2011. Robustness of intrinsic connectivity networks in the human brain to the presence of acoustic scanner noise. Neuroimage 55:1617-1632.

Langers DRM, van Dijk P, Backes WH. 2005. Lateralization, connectivity and plasticity in the human central auditory system. Neuroimage 28:490-499.

Leaver AM, Rauschecker JP. 2010. Cortical representation of natural complex sounds: effects of acoustic features and auditory object category. J Neurosci 30:7604-7612.

Leaver AM, Van Lare J, Zielinski B, Halpern AR, Rauschecker JP. 2009. Brain activation during anticipation of sound sequences. J Neurosci 29:2477-2485.

LeDoux JE, Farb C, Ruggiero DA. 1990. Topographic organization of neurons in the acoustic thalamus that project to the amygdala. J Neurosci 10:1043-1054.

Lowe MJ, Dzemidzic M, Lurito JT, Mathews VP, Phillips MD. 2000. Correlations in low-frequency BOLD fluctuations reflect cortico-cortical connections. Neuroimage 12:582-587. 
Marrelec G, Benali H. 2009. A theoretical investigation of the relationship between structural equation modeling and partial correlation in functional MRI effective connectivity. Comput Intell Neurosci 369341.

Marrelec G, Krainik A, Duffau H, Pélégrini-Issac M, Lehéricy S, Doyon J, Benali H. 2006. Partial correlation for functional brain interactivity investigation in functional MRI. Neuroimage 32:228-237.

Mazoyer B, Zago L, Mellet E, Bricogne S, Etard O, Houdé O, Crivello F, Joliot M, Petit L, Tzourio-Mazoyer N. 2001. Cortical networks for working memory and executive functions sustain the conscious resting state in man. Brain Res Bull 54:287-298.

Medford N, Critchley HD. 2010. Conjoint activity of anterior insular and anterior cingulate cortex: awareness and response. Brain Struct Funct 214:535-549.

Menon V, Uddin LQ. 2010. Saliency, switching, attention and control: a network model of insula function. Brain Struct Funct 214:655-667.

Mesulam MM. 1998. From sensation to cognition. Brain 121: 1013-1052.

Mhuircheartaigh RN, Rosenorn-Lanng D, Wise R, Jbabdi S, Rogers R, Tracey I. 2010. Cortical and subcortical connectivity changes during decreasing levels of consciousness in humans: a functional magnetic resonance imaging study using propofol. J Neurosci 30:9095-9102.

Moritz CH, Rogers BP, Meyerand ME. 2003. Power spectrum ranked independent component analysis of a periodic fMRI complex motor paradigm. Hum Brain Mapp 18:111-122.

Mutschler I, Wieckhorst B, Speck O, Schulze-Bonhage A, Hennig J, Seifritz E, Ball T. 2010. Time scales of auditory habituation in the amygdala and cerebral cortex. Cereb Cortex 20:2531-2539.

Nelken I. 2004. Processing of complex stimuli and natural scenes in the auditory cortex. Curr Opin Neurobiol 14:474-480.

Newman JD, Lindsley DF. 1976. Single unit analysis of auditory processing in squirrel monkey frontal cortex. Exp Brain Res 25:169-181.

Nieto Del Rincón PL. 2008. Autism: alterations in auditory perception. Rev Neurosci 19:61-78.

Phelps EA, LeDoux JE. 2005. Contributions of the amygdala to emotion processing: from animal models to human behavior. Neuron 48:175-187.

Poldrack RA, Clark J, Paré-Blagoev EJ, Shohamy D, Creso Moyano J, Myers C, Gluck MA. 2001. Interactive memory systems in the human brain. Nature 414:546-550.

Purdon PL, Weisskoff RM. 1998. Effect of temporal autocorrelation due to physiological noise and stimulus paradigm on voxel-level false-positive rates in fMRI. Hum Brain Mapp 6:239-249.

Raichle ME, Snyder AZ. 2007. A default mode of brain function: a brief history of an evolving idea. Neuroimage 37:1083-1090; discussion 1097-1099.

Rauschecker JP, Leaver AM, Mühlau M. 2010. Tuning out the noise: limbic-auditory interactions in tinnitus. Neuron 66: 819-826.

Rauschecker JP, Tian B. 2000. Mechanisms and streams for processing of "what" and "where" in auditory cortex. Proc Natl Acad Sci U S A 97:11800-11806.

Romanski LM, Tian B, Fritz J, Mishkin M, Goldman-Rakic PS, Rauschecker JP. 1999. Dual streams of auditory afferents target multiple domains in the primate prefrontal cortex. Nat Neurosci 2:1131-1136.
Rosell A, Giménez-Amaya JM. 1999. Anatomical re-evaluation of the corticostriatal projections to the caudate nucleus: a retrograde labeling study in the cat. Neurosci Res 34:257-269.

Sadaghiani S, Hesselmann G, Kleinschmidt A. 2009. Distributed and antagonistic contributions of ongoing activity fluctuations to auditory stimulus detection. J Neurosci 29:1341013417.

Salvador R, Suckling J, Coleman MR, Pickard JD, Menon D, Bullmore E. 2005. Neurophysiological architecture of functional magnetic resonance images of human brain. Cereb Cortex 15:1332-1342.

Schöpf V, Kasess CH, Lanzenberger R, Fischmeister F, Windischberger C, Moser E. 2010. Fully exploratory network ICA (FENICA) on resting-state fMRI data. J Neurosci Methods 192:207-213.

Seeley WW, Menon V, Schatzberg AF, Keller J, Glover GH, Kenna H, Reiss AL, Greicius MD. 2007. Dissociable intrinsic connectivity networks for salience processing and executive control. J Neurosci 27:2349-2356.

Sigalovsky IS, Melcher JR. 2006. Effects of sound level on fMRI activation in human brainstem, thalamic and cortical centers. Hear Res 215:67-76.

Smith SM, Fox PT, Miller KL, Glahn DC, Fox PM, Mackay CE, Filippini N, Watkins KE, Toro R, Laird AR, et al. 2009. Correspondence of the brain's functional architecture during activation and rest. Proc Natl Acad Sci U S A 106:1304013045.

Sridharan D, Levitin DJ, Menon V. 2008. A critical role for the right fronto-insular cortex in switching between central-executive and default-mode networks. Proc Natl Acad Sci U S A 105:12569-12574.

Talavage TM, Edmister WB, Ledden PJ, Weisskoff RM. 1999. Quantitative assessment of auditory cortex responses induced by imager acoustic noise. Hum Brain Mapp 7:79-88.

Uddin LQ, Menon V. 2009. The anterior insula in autism: underconnected and under-examined. Neurosci Biobehav Rev 33:1198-1203.

Williamson P. 2007. Are anticorrelated networks in the brain relevant to schizophrenia? Schizophr Bull 33:994-1003.

Wilson CL, Babb TL, Halgren E, Wang ML, Crandall PH. 1984. Habituation of human limbic neuronal response to sensory stimulation. Exp Neurol 84:74-97.

Winer JA, Lee CC. 2007. The distributed auditory cortex. Hear Res 229:3-13.

Worsley KJ, Friston KJ. 1995. Analysis of fMRI time-series revisited-again. Neuroimage 2:173-181.

Yetkin FZ, Roland PS, Mendelsohn DB, Purdy PD. 2004. Functional magnetic resonance imaging of activation in subcortical auditory pathway. Laryngoscope 114:96-101.

Zatorre RJ, Bouffard M, Belin P. 2004. Sensitivity to auditory object features in human temporal neocortex. J Neurosci 24:3637-3642.

Address correspondence to: Dave R.M. Langers Department of Otorhinolaryngology University Medical Center Groningen P.O. Box 30.001 9700 RB Groningen The Netherlands

E-mail: d.r.m.langers@umcg.nl 